\title{
Innovative Collaborative Practice to Optimize Pharmacotherapy for Frail Older Patients
}

\author{
Shanna Trenaman, Susan K Bowles, Laurie Mallery, Katalin Koller, and Melissa Andrew
}

\section{INTRODUCTION}

$\mathrm{M}$ edication use among older persons presents a double-edged sword. Pharmacotherapy is an important component in the management of chronic disease, and it can reduce morbidity and mortality. ${ }^{1-3}$ At the same time, adverse drug events are common in this population, resulting in functional impairment and increasing morbidity, mortality, and health care costs. ${ }^{4-7}$

Reported rates of adverse drug events in older populations range from $10 \%$ to $30 \%$ for ambulatory and inpatients alike..$^{8-12}$ Inappropriate prescribing, as indicated by explicit criterion-based or implicit judgment-based measures, increases the occurrence of adverse drug events. ${ }^{9} 10,13-15$ Therefore, the identification of inappropriate prescribing is important, as it can allow avoidance of preventable adverse drug events and optimization of pharmacotherapy for the frail older individual.

Frail older adults have interacting medical and social problems that occur in conjunction with mobility issues and polypharmacy, a situation that can give rise to complex medical needs. ${ }^{16}$ Advanced age is associated with accumulation of multiple comorbidities, many of which are managed with medications; as a result, polypharmacy is exceedingly common in frail older adults. ${ }^{17}$ As the number of medications increases, so does the risk of drug-drug interactions and adverse drug events. Individuals with many medications tend to self-report poor health, are more likely to have cognitive impairment, and have lower functional status. ${ }^{18}$ Because polypharmacy is highly prevalent among older adults who are frail, pharmacists working with patients in this age group have an opportunity to take a leadership role in monitoring for adherence, efficacy, and toxic effects and in helping those who are frail to manage their medications effectively. ${ }^{19}$

Randomized controlled trials have shown that the involvement of pharmacists in the care of older persons not only improves the appropriateness of medication regimens but also reduces drug-related morbidity. For example, in the Senior Care Study there was a $17 \%$ absolute risk reduction in the use of inappropriate medications when a pharmacist was incorporated into a multidisciplinary geriatric assessment team. ${ }^{20}$ These data have been confirmed by subsequent studies showing similar improvements in medication appropriateness when drug use was evaluated by a clinical pharmacist. ${ }^{21,22}$ Furthermore, there was an absolute risk reduction in drug-related morbidity of $10 \%$ to $20 \%$, as measured by hospital readmissions over a 3-month period, when clinical pharmacists provided collaborative care to older patients. ${ }^{23-25}$ This finding translated into a number needed to treat of 5 to 10 ; that is, when a clinical pharmacist was fully integrated into the multidisciplinary team, 1 hospital readmission was avoided for every 5 to 10 older patients discharged.

A comprehensive geriatric assessment can be used to assess an older person's medical, psychosocial, functional, and cognitive resources and problems $s^{26}$ and is an integral part of geriatric medicine. This assessment is an evidence-based process, based on information from the patient and collateral sources, that assesses a person's medical conditions and corresponding medications to ensure that each medication is used for an appropriate indication. It also assesses cognition, mood, mobility (including balance and falls), bowel and bladder function, nutritional status, sensory function (especially vision and hearing), overall function (basic and instrumental activities of daily living), and social circumstances. ${ }^{27}$ Use of this assessment has significant effects in terms of improving mortality, living location, physical status, and cognitive status. ${ }^{27}$ Such assessments are typically carried out by nurses, nurse practitioners, medical residents, and physicians. ${ }^{28}$

This article describes a collaboration between a pharmacist and a team of rotating geriatricians in the performance of comprehensive geriatric assessments at a community hospital and the impact of such assessments on the appropriateness of pharmacotherapy used by the frail older patients seen through the collaborative service.

\section{PROGRAM DESCRIPTION}

The Capital District Health Authority in Halifax, Nova Scotia, expanded its geriatric medicine inpatient consult services 
to a 100-bed community hospital serving a population of about 120000 in its catchment area. At the time of the study, this community was home to a substantial number of older adults and senior citizens, who accounted for the majority of hospital admissions. Given the impact that clinical pharmacy services have been shown to have on the care of frail older adults (as summarized in the previous section), an innovation grant was secured to fund a clinical pharmacist for 5 days a week in a position fully dedicated to clinical practice focusing on frail older adults. The pharmacist assigned to this role (S.T.) had graduated from an accredited Bachelor of Science in Pharmacy program and had completed a general accredited residency in pharmacy practice, including a 1-month rotation in geriatric medicine. The grantfunded position was the pharmacist's first employment position after completing the undergraduate degree and residency. The residency training had prepared the pharmacist to work to the full scope of practice, incorporating cognitive, functional, and physical assessment into practice, along with the more traditional roles of medication assessment and management. The service offered by this person represented the first clinical pharmacy service made available at the study hospital; before this position was established, pharmacy services at the hospital had been limited to dispensing tasks and provision of general drug information.

At the same time as the innovation grant was being used to provide clinical pharmacy services for frail older adults, an existing geriatric medicine consult service was expanded to the community hospital. Through this expanded service, the health authority supplied a geriatrician to the hospital (multiple physicians for 2-month rotations), with the physician being on site 3 days per week.

Initially, the geriatric medicine consult service involved the geriatrician working mostly in isolation, and the clinical pharmacist accepted only specifically requested consults from the geriatrician, physicians, other pharmacists, and emergency department staff for assessment and management of actual and potential drug-related problems. Referrals to the clinical pharmacist were sent by secure fax, were given over the telephone, or were discussed in person with the requesting clinician; geriatric medicine referrals were sent by secure fax. Given space limitations at the hospital, the geriatrician and the clinical pharmacist shared office space, and the 2 services shared a secure fax machine for receipt of referrals.

The geriatrician initially had a geriatric assessor available 2.5 days a week to help with organizing consults, liaising with other allied health professionals, and collecting collateral information. This individual had been trained as an occupational therapist. Despite the support of the geriatric assessor, there was frequent need for discussion of complex patient cases and clinical sharing between the clinical pharmacist and the geriatrician. Over the first 5 months of the innovation grant and expanded geriatric consult service, a collaboration evolved between these 2 health care professionals. Patients referred to the geriatrician for consultation were first assessed by the clinical pharmacist, who carried out a comprehensive geriatric assessment, including a detailed medical history, with review of results from relevant diagnostic and laboratory tests and a medication history. A report from the inpatient physiotherapy department was used to assess the patient's mobility, and, when possible, the pharmacist walked with the patient to check for gait disturbance that could have a medical or drug-related cause. Orthostatic vital signs were obtained if considered appropriate on the basis of the patient's history. The pharmacist also performed a cognitive assessment, which included completion of the Mini-Mental Status Examination for all patients and the Montreal Cognitive Assessment, Frontal Assessment Battery, and Geriatric Depression Scale where appropriate. The pharmacist also collected a collateral history detailing social status, cognitive history, and functional status with regard to instrumental activities of daily living (finances, medication management, driving, cooking, cleaning) and basic activities of daily living (toileting, bathing, grooming, dressing, eating).

The clinical pharmacist then developed recommendations and reviewed the entire case with the geriatrician, sharing any recommendations. When the situation was thought to be urgent or when the referring physician made a specific request, the pharmacist wrote orders or requested laboratory or diagnostic imaging; however, as for any consulting service, verification by the attending physician was required. The geriatrician completed any further assessment required, performed a detailed physical examination, and then shared any follow-up information with the pharmacist. The pharmacist educated patients and their families about medications and any medical conditions that had been diagnosed by the geriatrician. Patients were seen at followup appointments as needed to ensure resolution of the condition or conditions that had led to the consult. Follow-up could be as often as daily or as infrequently as weekly until discharge from the service.

The team continued to evolve, and eventually the geriatric assessor left the position and was not replaced. The pharmacist was seen as a highly valuable team member and, when funding for the innovation position ended, funding was organized to keep the pharmacist on the team. Thus, the geriatric medicine team became a collaboration between the geriatrician and the clinical pharmacist.

To assess the innovative pharmacist position for quality assurance purposes, data were collected for each referral reviewed by the pharmacist, including demographic variables such as age and sex, source of the referral, admission date, and discharge details (whether the patient went home, went to a long-term care facility, or died in hospital). The demographic data were collected as each consult was received by the pharmacist. There was interest in determining whether there was any improvement in medication regimens for patients who were seen by the pharmacist. 
Therefore, as part of the comprehensive geriatric assessment process, a detailed medication review was performed. The medications that a patient was taking at the time of first contact with the pharmacist were collected from the nursing medication administration record and were recorded as "initial medications". This list included both prescription medications and nonprescription products, such as vitamins, minerals, and natural health products. Interventions, if appropriate, were based on the recommendations of the clinical pharmacist or were determined in collaboration with the geriatrician. A patient's final medications were recorded at the last point of contact between the patient and the pharmacist before discharge from the service. A medication that could be used on an as-needed basis was included in the list of "final" medications only if the patient had received a dose while in hospital or if he or she had used the medication on an as-needed basis before the admission.

The mean numbers of medications at the initial assessment and final contact were calculated and compared to determine whether the pharmacist's interventions had significantly affected the number of medications used.

To quantify medication appropriateness, the medication appropriateness index (MAI) was used. The MAI is a scoring system to quantify drug appropriateness, in which higher scores correspond to greater inappropriateness of medication use, and which has been found to be a predictor of adverse drug reactions in older adults. ${ }^{29}$ For each medication in the patient's regimen, a series of 10 questions are asked, covering indication, effectiveness, dosage, correctness and practicality of directions for use, drugdrug and drug-disease interactions, possible duplication of therapy, duration, and cost. Each question is scored from 1 to 3 (where $1=$ appropriate, $2=$ marginally appropriate, and $3=$ not appropriate). The responses are weighted, and the resulting 10 values are summed to determine the MAI for each medication; the scores for all medications are then summed to generate a total MAI for the patient. Only those deemed inappropriate in some way get a score; appropriate statements receive no score under weighting. These calculations were performed as described previously. ${ }^{30}$ The lowest possible score for the MAI is zero; higher scores indicate more inappropriate prescribing. At the study hospital, the MAI was calculated for each patient's initial and final medication lists by the clinical pharmacist, who was intimately aware of the patient's medical conditions and medication needs. The initial medication list was based on medications at the time of the patient's first contact with the pharmacist, not the medications at the time of admission to hospital. The discharge MAI was based on information available up to the point of discharge from the pharmacist's care; any subsequent diagnoses or changes in medication use were not considered. Discharge from the pharmacist's care occurred when the reason for referral to the geriatric medicine service had been adequately assessed, treated, and resolved or when the patient was discharged from hospital or died. The average initial and final MAIs were calculated for all patients seen. These average initial and final MAIs were compared with a $t$ test to determine whether there was a statistically significant difference that might be attributable to the intervention of collaborative team. All calculations were completed in Microsoft Excel 2010 (Microsoft Corporation, Redmond, Washington).

\section{EVALUATION}

A total of 158 consults were reviewed by the pharmacist over the 7-month period of evaluation (August 2009 to March 2010). The average age of patients referred was 80.3 years. Although 95 (60.1\%) of the patients were able to return home, 37 (23.4\%) required placement in long-term care facilities, and 9 (5.7\%) died in hospital. The remaining 17 (10.8\%) of patients were still in hospital and being assessed periodically by the clinical pharmacist at the time of data analysis. In general, 6 new consults were completed by the pharmacist-geriatrician team each week, with follow-up appointments as necessary. The comprehensive geriatric assessment is an in-depth process, and each consult could take as long as 4 hours from initiation (with the pharmacist's review) to completion (once the formal report was written by the geriatrician).

In addition to the 17 patients who remained in hospital and under the pharmacist's care at the time of data analysis, data were unavailable for 5 patients who had been discharged but whose final medication list could not be accessed because of a delay in posting data to the electronic medical record. Therefore, data were available for analysis for 136 patients who were seen and discharged from the pharmacist's care over the 7-month period of evaluation. For these 136 patients, the mean number of medications declined from 10.3 to 9.8 (Table 1). The change in the number of medications was statistically significant $(p=0.005)$ but may not have been clinically important.

Table 1. Medication Changes during Pharmacist Assessment

\begin{tabular}{lccccc} 
& \multicolumn{2}{c}{ No. of Medications/Patient } & & \multicolumn{2}{c}{ MAl } \\
\cline { 2 - 3 } \cline { 5 - 6 } Timing & Mean \pm SD & Range & & Mean & Range \\
\hline Initial & $10.3 \pm 4.7$ & $25-2$ & & 10.72 & $83-0$ \\
Final & $9.8 \pm 4.2$ & $23-0$ & & 4.74 & $37-0$ \\
Change (initial - final) & Not calculated & NA & & -5.98 & NA \\
\cline { 2 - 3 } NA = not applicable, MAI = Medication Appropriateness Index, & \\
SD = standard deviation.
\end{tabular}


The overall MAI (sum of all weighted scores for each medication in a patient's medication regimen) declined from initiation to discharge, which represented a statistically significant improvement $(p<0.001)$ in medication appropriateness (Table $1)$. The breakdown of inappropriateness ratings are presented in Table 2. One limitation of the MAI is that it does not address omissions in therapy, which may be why medication appropriateness improved without a clinically important change in the number of medications. In particular, for many patients, new medications were initiated to treat previously untreated conditions.

The geriatrician group that collaborated with the pharmacist was confident in the pharmacist's work. It was possible to assign geriatricians to the rotation on a part-time basis, with the pharmacist providing continuity of care. The pharmacist worked to the full scope of practice, incorporating cognitive, functional, and physical assessment, along with medication assessment and management, in the care of frail older patients. This collaboration prevented repetition of assessments. It also allowed increased support for patients and their families, because the pharmacist was available regularly, even though the geriatrician was on site the equivalent of only 3 days per week.

\section{IMPLICATIONS AND SIGNIFICANCE FOR PRACTICE}

Collaboration between a clinical pharmacist with residency training in the care of elderly patients and a geriatrician at a Canadian community hospital led to an improvement in medication appropriateness that was both statistically significant and clinically meaningful. The innovative practice was based in a single centre and involved one clinical pharmacist working with a rotational supply of geriatricians. The collaboration reduced the amount of time that a geriatrician needed to be on site but did not compromise quality of care or continuity in the treatment plan.

For the purposes of quality assurance, the clinical pharmacist calculated the number of medications being taken at initial assessment and at discharge, as well as the MAI. The decrease in the number of medications was statistically significant $(p=0.005)$ but not clinically relevant. Medication appropriateness is an implicit measure that allows for consideration of patient-specific factors. All MAI values were calculated by one individual, the clinical pharmacist on the geriatric care team. The collaboration improved medication appropriateness as indicated by a decrease in the MAI $(p<0.001)$, but no comparator group was investigated. The MAI for older persons has been validated for both inter-rater and intra-rater validity. The overall inter-rater agreement for medication appropriateness had a kappa value of 0.83 , and the intra-rater agreement had an overall kappa value of $0.92 .{ }^{31}$ The inter-rater agreement for each of the individual MAI items was high for both appropriate and inappropriate
Table 2. Number of Medications Rated as Inappropriate for Each MAI Criterion*

\begin{tabular}{lrc} 
Criterion & $\begin{array}{c}\text { No. (\%) of Medications } \\
\text { Rated Inappropriate }\end{array}$ \\
\hline Indication & 109 & $(7.7)$ \\
Effectiveness & 119 & $(8.4)$ \\
Dosage & 94 & $(6.6)$ \\
Correct directions & 31 & $(2.2)$ \\
Practical directions & 34 & $(2.4)$ \\
Drug-drug interactions & 60 & $(4.2)$ \\
Drug-disease interactions & 188 & $(13.2)$ \\
Duplication & 89 & $(6.3)$ \\
Duration & 56 & $(3.9)$ \\
Cost & 29 & $(2.0)$ \\
\hline MA & &
\end{tabular}

MAI = Medication Appropriateness Index.

${ }^{*}$ Any value above zero was considered an inappropriate rating.

ratings, ranging from $80 \%$ to $100 \%$ (overall kappa $=0.64) .{ }^{31}$ Determination of baseline medication lists can be particularly challenging in geriatric medicine, although every effort was made to obtain the most accurate list possible for each patient seen by the clinical pharmacist in the program described here. In general, the medication administration record was used to identify baseline medications, so some of the most inappropriate medications would have been changed before the consultant pharmacist's assessment of the patient, and thus the admission medication list may have been even more inappropriate. As well, the clinical pharmacist was a consultant, and the attending physicians might not have implemented all recommended medication changes. The actual medications prescribed for each patient, not those recommended by the pharmacist and geriatrician, were used for the MAI calculations. All of these factors may have reduced the potential change in MAI.

The MAI criterion responsible for the most inappropriate ratings was related to drug-disease interactions (Table 2), in particular the use of excessive psychoactive medication in patients with cognitive impairment or delirium. Use of drugs lacking effectiveness or lacking an indication was also common. These findings likely reflect use of medications that were no longer necessary as conditions changed and people aged. For example, it was common to find individuals with low blood pressure remaining on antihypertensive medications that had probably been started many years previously, when hypertension had been a concern.

The finding that collaboration between a pharmacist and a geriatrician improved medication appropriateness but did not reduce the absolute number of medications has been previously reported. ${ }^{24}$ This result is of particular interest given the current focus on cost-saving initiatives. If the involvement of a pharmacist does not appreciably reduce the number of medications, administrators may wonder what benefits can be realized. The savings associated with removing 1 or 2 medications from a 
patient's medication regimen will result in some direct cost savings, but the real savings from pharmacist involvement in the care of frail older adults stems from the medication review, which can reduce hospital readmissions. Gillespie and other ${ }^{24}$ observed a $16 \%$ reduction in all visits to hospital over a 1-year follow-up period for patients who had undergone a pharmacist drug review in hospital, relative to those who did not undergo such a review. Applying this cost avoidance to the patient population served at the community hospital where the innovative practice was implemented and extrapolating from the 7-month period of analysis to a full calendar year (12 months) yielded a conservative estimate that pharmacist involvement in would have avoided a cost of $\$ 250000$ per year.

The improvement in MAI was in keeping with a previous study that showed a 5-point reduction in MAI with intervention from a pharmacist. ${ }^{32}$ No previous study has examined what a clinically meaningful reduction in the MAI would be, but previous work has shown that an increase in MAI is associated with worsening pain, an increase in emergency department visits, and an increase in hospital readmissions. ${ }^{23}$ These results suggest that improvement in the MAI would provide some degree of patient benefit.

A Royal College certified internist-geriatrician was available at the community hospital for the equivalent of 3 days per week to provide inpatient geriatric consult services. Collaborative work between the pharmacist and the geriatrician was helpful, as the lengthy comprehensive geriatric assessment was used by both the pharmacist and the geriatrician. Sharing information allowed more patients to be reviewed by the geriatrician and eliminated redundancy in the system by reducing repetition of work. Cost savings can be achieved by having a clinical pharmacist, rather than a geriatrician, perform the majority of the assessment and monitoring.

Details of the collaborative assessment were shared with the inpatient team but were not routinely forwarded to the family physician or the patient's community pharmacy. These are potential avenues for future consideration to encourage informationsharing.

\section{CONCLUSION}

This program evaluation has shown that a residency-trained pharmacist is in an ideal position to take on a collaborative role in the care of frail elderly patients. More specifically, in a collaborative environment, with regular involvement of a geriatrician, a residency-trained pharmacist trained in the tenets of geriatric medicine can organize and manage care for frail older individuals. In this setting, medication management may not have a clinically significant impact on the number of medications used but can significantly affect the appropriateness of medications used and, by extension, will ultimately prevent readmission to hospital.
References

1. Shepherd J, Blauw GJ, Murphy MB, Bollen EL, Buckley BM, Cobbe SM, et al. Pravastatin in elderly individuals at risk of vascular disease (PROSPER): a randomised controlled trial. Lancet. 2002;360(9346):1623-30.

2. Patel AB, Kostis JB, Wilson AC, Shea ML, Pressel SL, Davis BR. Long-term fatal outcomes in subjects with stroke or transient ischemic attack: fourteenyear follow-up of the systolic hypertension in the elderly program. Stroke. 2008;39(4):1084-9.

3. Beckett NS, Peters R, Fletcher AE, Staessen JA, Liu L, Dumitrascu D, et al. Treatment of hypertension in patients 80 years of age or older. NEngl J Med. 2008;358(18):1887-98.

4. Ensrud KE, Blackwell TL, Mangione CM, Bowman PJ, Whooley MA, Bauer DC, et al. Central nervous system-active medications and risk for falls in older women. J Am Geriatr Soc. 2002;50(10):1629-37.

5. Cao YJ, Mager DE, Simonsick EM, Hilmer SN, Ling SM, Windham BG, et al. Physical and cognitive performance and burden of anticholinergics, sedatives, and ACE inhibitors in older women. Clin Pharmacol Ther. 2008;83(3):422-9.

6. Fu AZ, Jiang JZ, Reeves JH, Fincham JE, Liu GG, Perri M 3rd. Potentially inappropriate medication use and healthcare expenditures in the US community-dwelling elderly. Med Care. 2007;45(5):472-6.

7. Fick DM, Mion LC, Beers MH L, Waller J. Health outcomes associated with potentially inappropriate medication use in older adults. Res Nurse Health. 2008;31(1):42-51.

8. Gray SL, Mahoney JE, Blough DK. Adverse drug events in elderly patients receiving home health services following hospital discharge. Ann Pharmacother. 199;33(11):1147-53.

9. Chang CM, Liu PY, Yang YH, Yang YC, Wu CF, Lu FH. Use of the Beers criteria to predict adverse drug reactions among first-visit elderly outpatients. Pharmacotherapy. 2005;25(6):831-8.

10. Onder G, Landi F, Liperoti R, Fialova D, Gambassi G, Bernabei R. Impact of inappropriate drug use among hospitalized older adults. Eur J Clin Pharmacol. 2005;61(5-6):453-9.

11. Hanlon JT, Pieper CF, Hajjar ER, Sloane RJ, Lindblad CI, Ruby CM, et al. Incidence and predictors of all and preventable adverse drug reactions in frail elderly persons after hospital stay. J Gerontol A Biol Sci Med Sci. 2006;61(5):511-5.

12. Chrischilles EA, VanGilder R, Wright K, Kelly M, Wallace RB. Inappropriate medication use as a risk factor for self-reported adverse drug effects in older adults. J Am Geriatr Soc. 2009;57(6):1000-6.

13. Laroche ML, Charmes JP, Nouaille Y, Picard N, Merle L. Is inappropriate medication use a major cause of adverse drug reactions in the elderly? $\mathrm{Br} J$ Clin Pharmacol. 2007;63(2):177-86.

14. Lund BC, Carnahan RM, Egge JA, Chrischilles EA, Kaboli PJ. Inappropriate prescribing predicts adverse drug events in older adults. Ann Pharmacother. 2010;44(6):957-63.

15. Page RL 2nd, Ruscin JM. The risk of adverse drug events and hospital-related morbidity and mortality among older adults with potentially inappropriate medication use. Am J Geriatr Pharmacother. 2006;4(4):297-305.

16. Rockwood K, Song X, MacKnight C, Bergman H, Hogan DB, McDowell I, et al. A global clinical measure of fitness and frailty in elderly people. CMAJ. 2005;173(5):489-95.

17. Colt HG, Shapiro AP. Drug-induced illness as a cause for admission to a community hospital. J Am Geriatr Soc. 1989;37(4):323-6.

18. Bressler R, Bahl JJ. Principles of drug therapy for the elderly patient. Mayo Clin Proc. 2003;78(12):1564-77.

19. Jyrkka J, Enlund H, Korhonen MJ, Sulkava R, Hartikainen S. Polypharmacy status as an indicator of mortality in an elderly population. Drugs Aging. 2009;26(12):1039-48.

20. Owens NJ, Sherburne NJ, Silliman RA, Fretwell MD. The Senior Care Study. The optimal use of medications in acutely ill older patients. $J \mathrm{Am}$ Geriatr Soc. 1990;38(10):1082-7.

21. Hanlon JT, Weinberger M, Samsa GP, Schmader KE, Uttech KM, Lewis IK, et al. A randomized, controlled trial of a clinical pharmacist intervention to improve inappropriate prescribing in elderly outpatients with polypharmacy. Am J Med. 1996;100(4):428-37. 
This single copy is for your personal, non-commercial use only.

For permission to reprint multiple copies or to order presentation-ready copies for distribution, contact CJHP at cjhpedit@cshp.ca

22. Burnett KM, Scott MG, Fleming GF, Clark CM, McElnay JC. Effects of an integrated medicines management program on medication appropriateness in hospitalized patients. Am J Health Syst Pharm. 2009;66(9):854-9.

23. Crotty M, Rowett D, Spurling L, Giles LC, Phillips PA. Does the addition of a pharmacist transition coordinator improve evidence-based medication management and health outcomes in older adults moving from the hospital to a long-term care facility? Results of a randomized, controlled trial. Am J Geriatr Pharmacother. 2004;2(4):257-64.

24. Gillespie U, Alassaad A, Henrohn D, Garmo H, Hammarlund-Udenaes M, Toss $\mathrm{H}$, et al. A comprehensive pharmacist intervention to reduce morbidity in patients 80 years or older: a randomized controlled trial. Arch Intern Med. 2009;169(9):894-900.

25. Makowsky MJ, Koshman SL, Midodzi WK, Tsuyuki RT. Capturing outcomes of clinical activities performed by a rounding pharmacist practicing in a team environment: the COLLABORATE study [NCT00351676]. Med Care. 2009; 47(6):642-50.

26. Jones DM, Song X, Rockwood K. Operationalizing a frailty index from a standardized comprehensive geriatric assessment. J Am Geriatr Soc. 2004; 52(11):1929-33.

27. Stuck AE, Siu AL, Wieland GD, Adams J, Rubenstein LZ. Comprehensive geriatric assessment: a meta-analysis of controlled trials. Lancet. 1993;342(8878):1032-6.

28. Welsh TJ, Gordon AL, Gladman JR. Comprehensive geriatric assessmenta guide for the non-specialist. Int J Clin Pract. 2014;68(3):290-3.

29. Hanlon, JT, Schmader, KE, Samsa, GP, Weinberger M, Uttech KM, Lewis $\mathrm{IK}$, et al. A method for assessing drug therapy appropriateness. J Clin Epidemiol. 1992;45(10):1045-51.

30. Hoti K, Sunderland B, Hughes J, Parsons R. An evaluation of Australian pharmacist's attitudes on expanding their prescribing role. Pharm World Sci. 2010;32(5):610-21.

31. Fitzgerald, LS, Hanlon, JT, Shelton, PS, Landsman PB, Schmader KE, Pulliam CC, et al. Reliability of a modified medication appropriateness index in ambulatory older persons. Ann Pharmacother. 1997;31(5):543-8.
32. O'Sullivan D, O'Mahony D, O'Connor MN, Gallagher P, Cullinan S, O'Sullivan R, et al. The impact of a structured pharmacist intervention on the appropriateness of prescribing in older hospitalized patients. Drugs Aging. 2014;31(6):471-81.

Shanna Trenaman, BScH, BScPharm, ACPR, is with the Department of Pharmacy, Capital District Health Authority, and the College of Pharmacy, Faculty of Health Professions, Dalhousie University, Halifax, Nova Scotia.

Susan K Bowles, PharmD, MSC, FCCP, is with the Department of Pharmacy, Capital District Health Authority, and the College of Pharmacy, Faculty of Health Professions, and the Division of Geriatric Medicine, Dalhousie University, Halifax, Nova Scotia.

Laurie Mallery, MD, FRCPC, is with the Division of Geriatric Medicine, Dalhousie University, Halifax, Nova Scotia.

Katalin Koller, MD, FRCPC, is with the Division of Geriatric Medicine, Dalhousie University, Halifax, Nova Scotia.

Melissa Andrew, MD, FRCPC, PhD, is with the Division of Geriatric Medicine, Dalhousie University, Halifax, Nova Scotia.

Competing interests: None declared.

\section{Address correspondence to:}

Shanna Trenaman

Department of Pharmacy

Dartmouth General Hospital

325 Pleasant Street - PO Box 1016

Dartmouth NS B2Y 4G8

e-mail: shanna.trenaman@dal.ca

\section{Acknowledgement}

Support for this study was provided by the Capital District Health Authority Innovation Fund. 